\title{
openheart Long-term survival after coronary bypass surgery and percutaneous coronary intervention
}

\author{
Per Mølstad, Rasmus Moer, Olaf Rødevand
}

To cite: Mølstad P, Moer R, Rødevand 0. Long-term survival after coronary bypass surgery and percutaneous coronary intervention. Open Heart 2016;3:e000489. doi:10.1136/openhrt-2016000489

Received 20 June 2016 Revised 9 September 2016 Accepted 20 September 2016

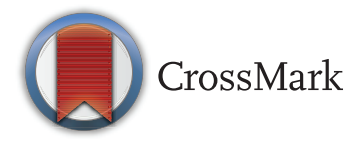

Department of cardiology, LHL Clinics Feiring, Feiring, Norway

Correspondence to Dr Per Mølstad; moelsta@online.no

\section{ABSTRACT}

Objectives: To assess whether there exists a longterm difference in survival after treatment with coronary bypass surgery or percutaneous coronary intervention in patients with coronary disease as judged by all-cause mortality.

Methods: Retrospective study from the Feiring Heart Clinic database of survival in 22880 patients-15 078 treated with percutaneous coronary intervention and 7802 with bypass surgery followed up to 16 years.

Results: Cox regression and propensity score analysis showed no difference in survival for one-vessel and two-vessel disease during the whole study period. In three-vessel disease, however, the analysis revealed a consistent and highly significant survival benefit in the first 8 years with an HR of $0.76(95 \% \mathrm{Cl} 0.69$ to 0.84 , $p<0.001)$ in favour of bypass surgery with similar survival rates in the two treatment strategies after that time period.

Conclusions: Treatment strategy did not affect survival in one-vessel and two-vessel disease, but bypass surgery offered an improved survival in the first 8 years in patients with three-vessel disease. These results are consistent with most previous reports and the survival benefit should be taken into account when selecting a strategy for this patient group.

\section{INTRODUCTION}

The optimal invasive treatment of coronary artery disease has been debated for years, ${ }^{1-8}$ and new arguments for both coronary artery bypass grafting (CABG) and percutaneous coronary intervention (PCI) have been put forward as technology and medication have improved. In general, most reports from both randomised and observational studies have indicated a survival benefit of CABG compared to PCI in subsets of patients with multivessel disease and complicated coronary pathology. ${ }^{13-6} 910$ Typically, the results after the introduction of drug-eluting stents (DES) and the further development of secondgeneration DES have been promoted as an argument in favour of treating more patient subsets with PCI instead of CABG. ${ }^{11}{ }^{12}$ However, reports have been published that

\section{KEY QUESTIONS}

What is already known about this subject?

- Both randomised and observational studies with a follow-up of at least 5 years indicate a survival benefit of coronary artery bypass grafting (CABG) treatment compared to percutaneous coronary intervention $(\mathrm{PCl})$ in patients with complex coronary artery disease.

What does this study add?

- Our study supports the survival benefit of CABG in patients with three-vessel disease but, in addition, indicates that this benefit is limited to the first 8 years after the index procedure. After that time period, the survival rates seem equal. Further, the study indicates that $\mathrm{PCl}$ and $\mathrm{CABG}$ have identical survival rates in one-vessel and two-vessel disease.

How might this impact on clinical practice?

- The study adds guidance to the process of selecting initial invasive treatment in patients with coronary artery disease.

question this claim, as the survival rate still is in favour of CABG, ${ }^{713}$ and has not changed after the introduction of DES. Inherent in adopting results from recent trials is the problem of limited time of follow-up. There is a paucity of studies reporting follow-up beyond 7-8 years and those who do have recruited their patients before year $2000 .{ }^{1} \mathrm{It}$ is reasonable to assume that the treatment modalities at that time will have limited impact on today's practice. The aim of the present study was to compare the long-term survival of patients initially allocated to PCI or CABG from 1999 until 2014 and followed up to 16 years.

\section{MATERIALS AND METHODS}

Feiring Heart Clinic has had a common database for the cardiological and surgical department since 1999. This database contains information on demographics, clinical and angiographic parameters, treatment, diagnosis 
(International Classification of Disease 10th Edition, ICD 10) and surgical operative codes (Nordic Medico-Statistical committee Classification of Surgical procedures, NCSP). Only patients submitted to angiography and the subsequent treatment at our institution were included in the analysis. The patients were recruited from March 1999 until December 2014. The survival status as of 20 September 2015 was established through the Norwegian National Registry, which also gave formal consent to obtain the data. The end point of the study was all-case mortality. Emigrated patients were censored at the date of emigration and constituted only $0.5 \%$ of the population. The treatment allocation was according to the strategy chosen at the first admission and only information from that admittance was used in the analysis. Thus, each patient could only enter the study once. Patients with a combined operation with valves and bypass were excluded from the analyses.

\section{Statistical analysis}

The purpose of the analysis was to compare the survival rates of patients treated with PCI and CABG on an intention-to-treat basis. A separate analysis was performed on a per-protocol basis with patients operated with CABG within 30 days of their initial PCI treatment.

Continuous variables were tested for normality with the skewness and kurtosis test and, if deviating from normality, tested with the Kruskal-Wallis test. Variables with normal distribution were evaluated with analysis of variance. Categorical variables were tested with Fisher's exact test or $\chi^{2}$ test in case of excessive permutations.

Univariate survival analyses were performed using the Kaplan-Meier estimator and log-rank test. In the multivariable survival analysis, missing values for continuous variables were imputed by a best subset multiple regression and categorical variables were imputed to the most frequent subset. The results of the analyses were also confirmed by the method of multiple imputation.

Multivariable survival analysis accounting for the differences at baseline were performed with Cox proportional hazard regression. The model was built by a forward selection process. Continuous variables were tested for linearity in $\log$ hazard by quartile plots. Interactions were kept in the model if they were biologically interesting and statistically significant. The proportional hazard assumptions were evaluated by a test based on Schoenfeld residuals, by $\log -\log$ plots and by interaction with time and time-split at different points of time. The final model was tested with linktest and plotting Cox-Snell residuals versus the Nelson-Aalen estimator of cumulative hazard for evaluating goodness-of-fit. In case of violation of proportional hazard in important covariates pertaining to these analyses, a landmark analysis would be performed. The log-likelihood for models split into two time intervals $(\leq \mathrm{t}$ and $>\mathrm{t})$ was calculated for each year. The model with the highest log-likelihood was considered the most appropriate model to use.

Selection bias was addressed by propensity score analysis. A logit model was built from baseline variables predicting treatment allocation $(\mathrm{PCI}=0, \quad \mathrm{CABG}=1)$. Continuous variables were checked for linearity in logit. All significant variables and interaction were kept in the model that was tested for goodness-of-fit by the Hosmer and Lemeshow test. From the model, the c-statistic was calculated (area under the receiver operating (ROC) curve). The propensity scores were calculated from the logit model. The scores were used as a single adjusting covariate in a Cox regression and the logit of the propensity score was used for 1:1 matching without replacement and a caliper width of 0.2 times the SD of the logit of the propensity score. ${ }^{14}$ The matched pairs were then used in a Cox regression stratified on pairs. In all Cox regressions, the robust version of calculating SEs was employed.

The effect of an unmeasured binary confounder on the HR for the treatment effect from the Cox model was evaluated using the method of Lin et al. ${ }^{15}$

All analyses were performed in STATA V.14 (College Station, Texas, USA), and the propensity matching with the STATA program psmatch2.

\section{RESULTS}

A total of 22880 patients were eligible for the analysis with known survival status on 20 September 2015, of whom 15078 were treated with PCI and 7802 with CABG. The study end point was all-cause mortality and was encountered in 5408 patients. The total time at risk was 177371 patient years in the whole population with 114115 years in the PCI cohort and 63256 years in the CABG treatment group. The median time at risk was 7.2 years for the PCI group and 7.9 years for the CABG group.

Baseline demographics, clinical and angiographical data are given in table 1.

The Kaplan-Meier plot of the unadjusted mortality according to treatment strategy is shown in figure 1 . From table 1, it is evident that the cohorts have different values for many covariates expected to affect survival. Typically, the surgical cohort is older and has general arteriosclerosis, diabetes and three-vessel disease more frequently.

The variables from table 1 were tested for inclusion in a multivariable Cox model by a forward selection process. The final model contained 13 main effects and one interaction. In fact, a number of interactions were statistically significant, but the only interesting one pertaining to these analyses was the interaction between the number of the diseased vessel and strategy. The other significant interactions had a minimal effect on the other covariates and were not interesting for the present analysis. A Kaplan-Meier plot of mortality in the two strategies for one-vessel, two-vessel and three vessel disease is shown in figure 2. The linktest for the final model was negative and a plot of Cox-Snell residuals versus the Nelson-Aalen estimator indicated a reasonable goodness-of-fit. Proportional hazard assumption was 
Table 1 Baseline demographic and clinical variables

\begin{tabular}{|c|c|c|c|}
\hline Variable & $\begin{array}{l}\mathrm{PCl} \\
\mathrm{N}=15078\end{array}$ & $\begin{array}{l}\text { CABG } \\
\mathrm{N}=7802 \\
\end{array}$ & p Value \\
\hline Age years $($ mean $\pm S D)$ & $65 \pm 11$ & $67 \pm 10$ & $<0.001$ \\
\hline Gender (male/female) \% & 73.0/27.0 & $78.2 / 21.8$ & $<0.001$ \\
\hline Ejection fraction \% (mean $\pm S D)$ & $68 \pm 12$ & $67 \pm 12$ & 0.04 \\
\hline LVEDP mm Hg (mean $\pm S D)$ & $14.1 \pm 4.8$ & $15.3 \pm 5.9$ & $<0.001$ \\
\hline Generalised arteriosclerosis \% (number) & $4.6(696)$ & $7.0(548)$ & $<0.001$ \\
\hline Other significant disease \% (number) & $6.2(933)$ & $5.6(438)$ & 0.08 \\
\hline Exercise ECG \% (number) & & & $<0.001$ \\
\hline Not performed & $37.4(5281)$ & $29.2(2155)$ & \\
\hline Negative exercise test & $9.9(1395)$ & $6.0(444)$ & \\
\hline Inconclusive result & $25.1(3536)$ & $20.5(1511)$ & \\
\hline Ischaemic exercise response & 27.7 (3904) & 44.4 (3279) & \\
\hline \multicolumn{4}{|l|}{ CCS function class $\%$ (number) } \\
\hline 0 & $1.9(254)$ & $1.9(137)$ & \\
\hline I & $5.9(809)$ & $4.7(336)$ & \\
\hline II & $37.7(5143)$ & $33.5(2374)$ & \\
\hline III & 40.5 (5519) & $46.8(3316)$ & \\
\hline IV & $14.1(1917)$ & $13.0(924)$ & $<0.001$ \\
\hline Unstable angina \% (number) & $29.3(4416)$ & $27.0(2106)$ & $<0.001$ \\
\hline Diabetes \% (number) & $13.3(1998)$ & $16.4(1278)$ & $<0.001$ \\
\hline Hypertension \% (number) & 32.5 (4904) & $36.5(2845)$ & $<0.001$ \\
\hline Current smoker \% (number) & $21.6(3253)$ & $19.4(1512)$ & $<0.001$ \\
\hline Previous myocardial infarction \% (number) & $36.3(5450)$ & 36.5 (2843) & 0.68 \\
\hline Previous $\mathrm{PCl} \%$ (number) & $17.0(2549)$ & $8.4(655)$ & $<0.001$ \\
\hline Previous CABG \% (number) & $12.5(1878)$ & $2.1(163)$ & $<0.001$ \\
\hline Radial entry site \% (number) & $84.2(12701)$ & $82.4(6430)$ & $<0.001$ \\
\hline \multicolumn{4}{|l|}{ Coronary angiography } \\
\hline One-vessel disease \% (number) & 46.2 (7029) & $2.2(175)$ & \\
\hline Two-vessel disease \% (number) & 30.1 (4534) & $17.9(1400)$ & \\
\hline Three-vessel disease \% (number) & $23.3(3515)$ & $79.8(6227)$ & $<0.001$ \\
\hline
\end{tabular}

Generalised arteriosclerosis is defined as previous known extra-cardiac arteriosclerotic symptoms. CCS, Canadian Cardiovascular Society functional class for angina. Diabetes is defined as previously known and treated with diet or drugs. Other significant disease is defined as renal, hepatic or pulmonary disease and serious obesity deemed of importance in the treatment at the discretion of the physician. Unstable angina also includes patients with non ST-elevation myocardial infarction.

EECG=exercise ECG; LVEDP, left ventricular end diastolic pressure.

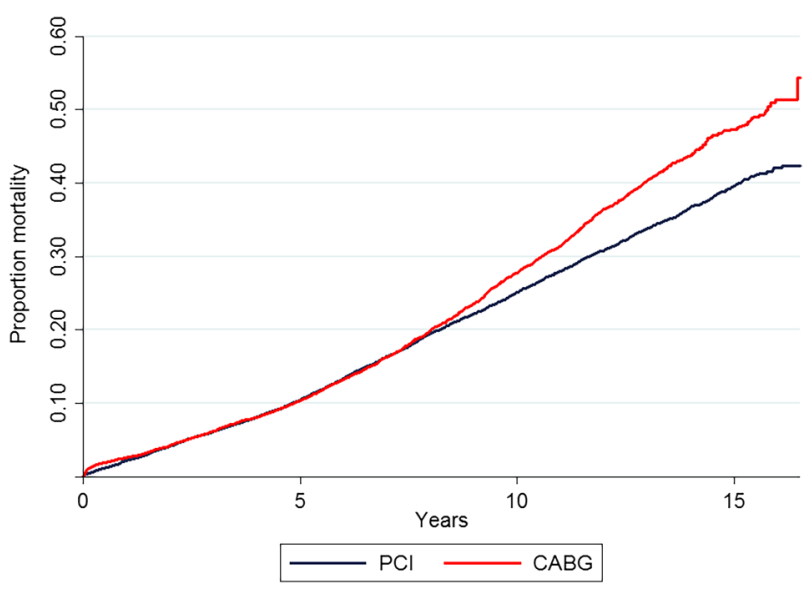

Figure 1 Kaplan-Meier estimate of mortality in the two treatment strategies with significant difference between the two groups (log-rank test $<0.001$ ) CABG, coronary artery bypass grafting; $\mathrm{PCl}$, percutaneous coronary intervention.

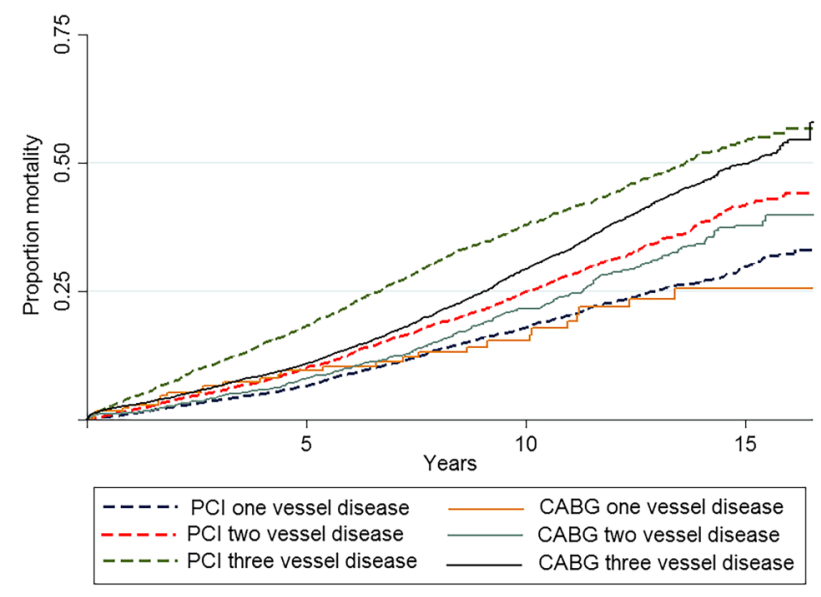

Figure 2 Kaplan-Meier estimate of mortality in the two treatment strategies divided in number of diseased vessels. There is a significant difference between the groups (log-rank test $<0.001)$. CABG, coronary artery bypass grafting; $\mathrm{PCl}$, percutaneous coronary intervention. 
Table 2 Cox regression model up to 8 years follow-up

\begin{tabular}{|c|c|c|c|}
\hline Variable & HR & $95 \% \mathrm{Cl}$ & p Value \\
\hline Age/5 years & 1.53 & 1.50 to 1.56 & $<0.001$ \\
\hline Ejection fraction $/ 5 \%$ & 0.88 & 0.86 to 0.89 & $<0.001$ \\
\hline Other significant disease & 2.01 & 1.79 to 2.27 & $<0.001$ \\
\hline Smoking & 1.58 & 1.44 to 1.73 & $<0.001$ \\
\hline Diabetes & 1.41 & 1.30 to 1.54 & $<0.001$ \\
\hline Previous CABG & 1.12 & 1.00 to 1.25 & 0.047 \\
\hline \multicolumn{4}{|l|}{ Exercise ECG* } \\
\hline Negative exercise test & 0.69 & 0.59 to 0.81 & $<0.001$ \\
\hline Inconclusive result & 0.79 & 0.72 to 0.87 & $<0.001$ \\
\hline Ischaemic exercise response & 0.67 & 0.61 to 0.73 & $<0.001$ \\
\hline CCS function class 3 or $4 \dagger$ & 1.31 & 1.21 to 1.42 & $<0.001$ \\
\hline Number of diseased coronary arteries $\ddagger$ & 1.19 & 1.13 to 1.26 & $<0.001$ \\
\hline Presence of generalised arteriosclerosis & 1.35 & 1.19 to 1.53 & $<0.001$ \\
\hline Gender & 1.14 & 1.06 to 1.23 & 0.001 \\
\hline Previous myocardial infarction & 1.10 & 1.02 to 1.18 & 0.014 \\
\hline Treatment strategy§ & 1.29 & 0.86 to 1.93 & 0.23 \\
\hline Interaction term strategy and number of diseased vessel & 0.84 & 0.72 to 0.97 & 0.02 \\
\hline
\end{tabular}

tested with the method of Schoenfeld residuals and indicated that a number of covariates actually were not proportional in hazard. They were then evaluated by plotting the scaled Schoenfeld residuals versus time with LOcally-WEighted Scatter-plot Smoothing (LOWESS). The variables were also used as stratification variables. Both methods indicated that the violations of assumptions were minimal with no impact on the parameters of interest and could therefore be kept in the model as covariates. Those HRs can be viewed as an average over time. ${ }^{16}$ The only exception was the covariate treatment strategy where all methods (Schoenfeld residuals, log-log plots, interaction with time and time-split) indicated violation of proportional hazard and where the results of interest were very different without taking the violation into account. Cox models with all the covariates were then run with time split at each different year and the model with the highest log-likelihood was found by splitting time at 8 years. Landmark analyses were then performed in the two time periods before and after 8 years. Treatment strategy was proportional in hazard within each of the two time periods. In the first 8 years, the interaction between strategy and number of diseased vessels was significant $(p=0.02$, likelihood ratio test between the models), but not after 8 years $(p=0.47)$. All analysis in the first 8 years therefore include the interaction term (table 2).

The HRs for the first 8 years in the number of diseased vessels are given in table 3 .

There is no difference in survival for one-vessel and two-vessel disease, but a highly significant difference in three-vessel disease with an HR of 0.76 (95\% CI 0.69 to $0.84, \mathrm{p}<0.001)$ as depicted in figure 3 and table 3 . The
Table 3 HRs in the first 8 years for one-vessel two-vessel and three vessel disease

\begin{tabular}{lllc}
\hline Variable & HR & 95\% Cl & p Value \\
\hline One-vessel disease & 1.08 & 0.83 to 1.40 & 0.57 \\
Two-vessel disease & 0.91 & 0.79 to 1.04 & 0.16 \\
Three-vessel disease & 0.76 & 0.69 to 0.84 & $<0.001$ \\
\hline
\end{tabular}

results for three-vessel disease was evaluated for unmeasured confounders by the method of Lin et al. ${ }^{15}$ The HR of this treatment effect could be reduced to a nonsignificant level if an unmeasured binary confounder existed with an HR of 3.0 in both groups and a difference in prevalence of $20 \%$ between the treatment options, or with a difference in prevalence of $30 \%$ if the common HR was 2.0.

The same Cox model after 8 years revealed no difference between the treatment strategies with an $\mathrm{HR}=1.07$ (95\% CI 0.94 to $1.20, \mathrm{p}=0.29$ ). Performing separate analyses for one-vessel, two-vessel and three-vessel disease gave virtual identical results, as did the exclusion of patients with a previous CABG.

The use of multiple imputation of missing data yielded identical results. There were 206 patients with PCI who were treated with CABG within 30 days after the initial PCI treatment. A separate Cox regression as a per protocol analysis yielded similar results as the intention-to-treat analysis.

The final logit model for estimation of propensity scores contained 16 main effects and 24 interactions. The continuous variables were modelled as fractional polynomials. The Hosmer and Lemeshow test for 


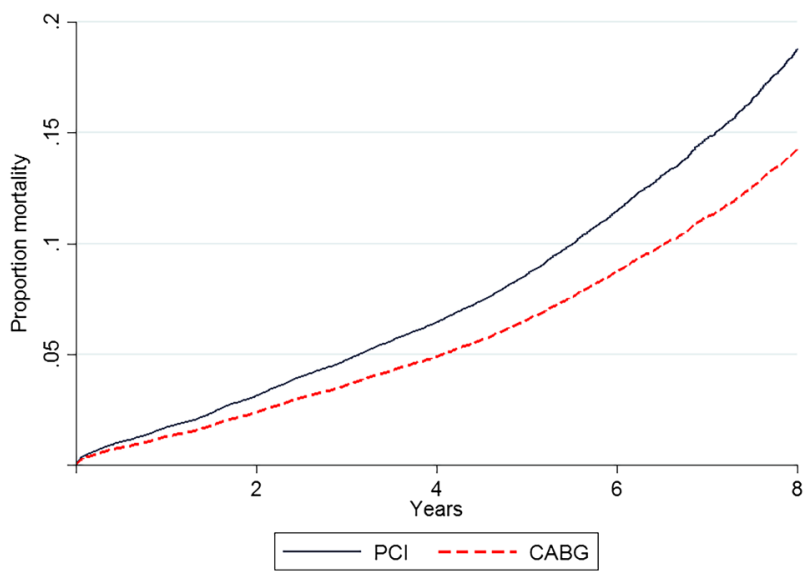

Figure 3 Cox regression of proportion mortality in the first 8 years for patients with three-vessel disease with a significant difference between the treatment strategies $(\mathrm{p}<0.001)$. CABG, coronary artery bypass grafting; $\mathrm{PCl}$, percutaneous coronary intervention.

goodness of fit was negative $(\mathrm{p}=0.65)$ and c-statistics (area under the ROC curve) 0.904. The propensity scores were calculated from this model. For the cohorts, the propensity scores were $($ mean $\pm \mathrm{SD}$ ) $0.18 \pm 0.23$ (range $0.0008-0.96$ ) for PCI and $0.66 \pm 0.25$ (range 0.006-1.0) for $\mathrm{CABG}$.

The propensity score analyses were performed separately before and after 8 years. The Cox model for the first 8 years with only propensity score and strategy as covariates revealed a strong and significant interaction $(\mathrm{p}<0.001)$. The score was therefore divided at 0.5 and two separate Cox regressions made and the interaction was then no longer significant. However, in those models, the propensity scores were not proportional in hazard and stratified analyses were performed on quintiles of the propensity score. In propensity score $>0.5$, the $\mathrm{HR}=0.87$ ( $95 \%$ CI 0.77 to $0.98, \mathrm{p}=0.02)$ and in propensity score $<0.5$ the HR=1.04 (95\% CI 0.91 to 1.19 , $\mathrm{p}=0.58)$.

After 8 years, the interaction between treatment strategy and propensity score was not significant $(p=0.23)$ and there was no violation of the proportional hazard assumption. The HR was marginally significant in favour of PCI (HR=1.15, 95\% CI 1.01 to $1.30, \mathrm{p}=0.04)$.

The propensity scores were also used in matching analyses before and after 8 years. Separate analyses were performed in propensity score above and below 0.5 for the first 8 years. In propensity score above 0.5 , there were 1798 matched pairs and the absolute standardised \% bias was reduced from $($ mean \pm SD) $9.2 \pm 7.4$ to $4.4 \pm 3.3$. The Cox analysis stratified on matched pairs revealed an HR in favour of CABG of 0.81 (95\% CI 0.728 to 0.92 , $\mathrm{p}=0.001$ ). A Kaplan-Meier mortality plot of matched pair during the whole follow-up period is shown in figure 4 . In propensity score $<0.5$, there were 1794 matched pairs with a reduction in \% bias from (mean $\pm \mathrm{SD}$ ) 10.6 \pm 17.5

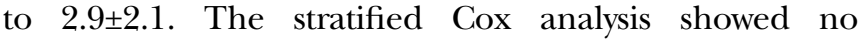

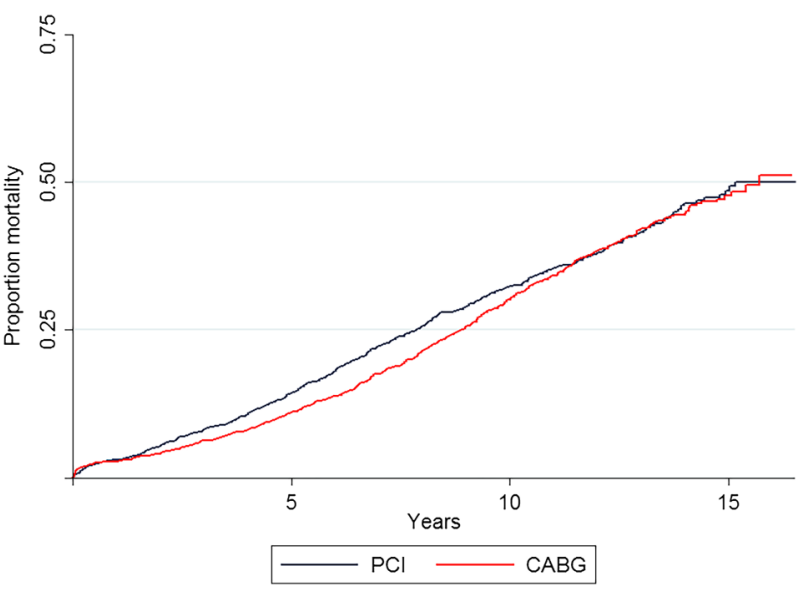

Figure 4 Kaplan-Meier estimate of mortality in 1798 propensity score matched pairs with a propensity score $>0.5$ for the whole observational period. Log-rank test stratified on matched pairs indicates the difference to be of borderline significance $(p=0.059)$. CABG, coronary artery bypass grafting; $\mathrm{PCl}$, percutaneous coronary intervention.

difference between the treatment strategies with an HR of 0.96 (95\% CI 0.84 to $1.10, \mathrm{p}=0.55)$. In the matching after 8 years, there were 1677 matched pairs with a reduction in $\%$ bias from $($ mean \pm SD) $20.1 \pm 34.9$ to 5.7 \pm 4.2 . The stratified Cox regression revealed an HR of $1.12(95 \%$ CI 0.97 to $1.28, \mathrm{p}=0.11)$.

\section{DISCUSSION}

The selection of revascularisation strategy in patients with coronary artery disease has been debated for years, and the continuous development of new drugs and medical technology has made the optimal strategy a moving target. Most studies suffer from the fact that the follow-up period has been limited (in most cases 5 years or less). Our study elucidates the results of a long-term follow-up of all-cause mortality with a median possible observation period (provided no deaths) of 9.3 years (IQR 5.3-12.9 years) from time of entry to end of study. The results from the analyses indicated that the effect of treatment might have a different impact on mortality during the elapse of time, and that the performance of separate analyses before and after 8 years of follow-up was justified. In the ordinary Cox regression during the first 8 years, the treatment selection did not affect survival for one-vessel and two-vessel disease, but CABG had a clear survival benefit in patients with three-vessel disease with an HR of 0.76 (95\% CI 0.69 to 0.84 , $\mathrm{p}<0.001)$. The propensity score analysis support this result. As can be expected, there is a strong correlation between the propensity score and number of diseased vessels. Three-vessel disease was present in $96.6 \%$ of the patients with a propensity score $>0.5$. The results of the ordinary Cox regression and propensity score analyses are therefore consistent in that survival is improved for patients with CABG with three-vessel disease in the first 
8 years with no further difference after that time. This time limited effect of CABG can also be inferred from figures 2 and 4.

A sensitivity analysis using the method indicated by Lin $e t a l^{15}$ evaluates the possible influence of an unmeasured binary confounder on these results. An example of such a confounder could be patient frailty as suggested by Weintraub et al. ${ }^{8}$ The sensitivity analysis indicate that a skewed distribution of a single strong confounder or several confounders acting in concert could conceivably account for the observed difference in survival. The existence of such confounder(s), however, is probably not very likely taking into account the number of baseline differences accounted for in the analysis.

The present results are in agreement with previous reports from our database, ${ }^{6} 7$ where the follow-up was ended after 5 years. They are also corroborated by the 5 -year follow-up of the randomised Syntax study ${ }^{2} 17$ and a number of observational studies, reviews and meta-analysis, ${ }^{1}$ 3-5 810131819 although not all reports agree on improved survival with CABG. ${ }^{12}{ }^{20}$ Those who do not agree either have a short follow-up time (mean 2.9 years ${ }^{12}$ or include a substantial amount of one-vessel and two-vessel disease in their analyses. ${ }^{20}$ Thus, it seems fair to conclude that the majority of studies and evidence indicate a moderate but significant survival benefit of CABG over PCI in patients with three-vessel disease.

We have previously described a consistent survival benefit of CABG compared to PCI before and after the introduction of DES, ${ }^{7}$ an observation shared by others. ${ }^{13}$ Whether this also will be the case after the introduction of second-generation DES is at present not known.

The other interesting aspect with the present results is the time limit of the improved survival. The data indicate a survival benefit of treatment selection in the first 8 years and no further difference in survival rates after that point of time. Of course, an improved survival will always vanish if the cohorts are followed long enough, as eventually all participants in a study will die. There are at least two aspects in this connection that deserve mention. First of all, the age of the remaining population after 8 years of follow-up is quite high with a median age of 71 years and $25 \%$ of the population more than 79 years old. In this age group, it is reasonable to assume that other causes of death than coronary disease might be prevalent and thereby dilute the effect of treatment strategy. Second, after 8 years, the preponderance of degenerated vein graft might affect survival in the CABG group. Studies have shown a limited long-term patency of vein-grafts with $75-86 \%$ open after 5 years ${ }^{21-23}$ and $61 \%$ after 10 years. ${ }^{24}$ Interestingly, Fitzgibbon et $a l^{21}$ also claim to observe an increase in the mortality rate after 7 years in a CABG-treated population.

There are several limitations to this study. First of all, the fact that it is an observational study where a selection bias never can be completely ruled out. However, the propensity score analyses addressing this problem are consistent with the Cox regression analyses, and previous reports both from observational and randomised studies are largely supportive of these results. ${ }^{1-5} 810131819$ Even though the sensitivity analysis indicate that the observed differences could be explained by the existence of a strong unobserved confounder or several unobserved confounders acting in concert, such confounders could, however, also increase the differences depending on the distribution between the treatment strategies. Second, the information of angiographic results are not detailed enough in the database to calculate the Syntax score, ${ }^{25}$ preventing the possibility of a better classification of complex coronary pathology. Finally, multiple comparisons without Bonferroni adjustments justify a cautious interpretation of the reported $p$ values.

In conclusion, our study indicates that a moderate survival benefit of CABG over PCI in patients with threevessel disease exists in the first 8 years after the procedure with no important difference in the survival rates after that time.

Competing interests None declared.

Ethics approval Norwegian National Registry.

Provenance and peer review Not commissioned; externally peer reviewed.

Data sharing statement No additional data are available.

Open Access This is an Open Access article distributed in accordance with the Creative Commons Attribution Non Commercial (CC BY-NC 4.0) license, which permits others to distribute, remix, adapt, build upon this work noncommercially, and license their derivative works on different terms, provided the original work is properly cited and the use is non-commercial. See: http:// creativecommons.org/licenses/by-nc/4.0/

\section{REFERENCES}

1. Smith PK, Califf RM, Tuttle RH, et al. Selection of surgical or percutaneous coronary intervention provides differential longevity benefit. Ann Thorac Surg 2006;82:1420-9.

2. Mohr FW, Morice MC, Kappetein AP, et al. Coronary artery bypass graft surgery versus percutaneous coronary intervention in patients with three-vessel disease and left main coronary disease: 5-year follow-up of the randomised, clinical SYNTAX trial. Lancet 2013;381:629-38.

3. Malenka DJ, Leavitt BJ, Hearne MJ, et al. Comparing long-term survival of patients with multivessel coronary disease after CABG or PCI: analysis of BARI-like patients in Northern New England. Circulation 2005;112(Suppl 9):1371-6.

4. Hannan EL, Wu C, Walford G, et al. Drug-eluting stents vs. coronary-artery bypass grafting in multivessel coronary disease. N Engl J Med 2008;358:331-41.

5. Hannan EL, Racz MJ, Walford G, et al. Long-term outcomes of coronary-artery bypass grafting versus stent implantation. $N$ Engl J Med 2005;352:2174-83.

6. Mølstad P. Survival after percutaneous coronary intervention and coronary artery bypass grafting in a single centre. Scand Cardiovasc J 2007;41:214-20.

7. Mølstad P. Survival difference between coronary bypass surgery and percutaneous coronary intervention. Scand Cardiovasc $J$ 2015;49:177-82.

8. Weintraub WS, Grau-Sepulveda MV, Weiss JM, et al. Comparative effectiveness of revascularization strategies. $N$ Engl J Med 2012;366:1467-76.

9. Deb S, Wijeysundera HC, Ko DT, et al. Coronary artery bypass graft surgery vs percutaneous interventions in coronary revascularization: a systematic review. JAMA 2013;310:2086-95.

10. Hlatky MA, Boothroyd DB, Bravata DM, et al. Coronary artery bypass surgery compared with percutaneous coronary interventions 
for multivessel disease: a collaborative analysis of individual patient data from ten randomised trials. Lancet 2009;373:1190-7.

11. Harrington RA. Selecting revascularization strategies in patients with coronary disease. N Engl J Med 2015;372:1261-3.

12. Bangalore S, Guo Y, Samadashvili Z, et al. Everolimus-eluting stents or bypass surgery for multivessel coronary disease. $N$ Engl $J$ Med 2015;372:1213-22.

13. Hlatky MA, Boothroyd DB, Baker LC, et al. Impact of drug-eluting stents on the comparative effectiveness of coronary artery bypass surgery and percutaneous coronary intervention. Am Heart $J$ 2015;169:149-54.

14. Austin PC. Optimal caliper widths for propensity-score matching when estimating differences in means and differences in proportions in observational studies. Pharmaceut Statist 2011;10:150-61.

15. Lin DY, Psaty BM, Kronmal RA. Assessing the sensitivity of regression results to unmeasured confounders in observational studies. Biometrics 1998;54:948-63.

16. Therneau T, Grambsch P. Modeling survival data: extending the cox model. New York: Springer, 2000.

17. Head SJ, Davierwala PM, Serruys PW, et al. Coronary artery bypass grafting vs. percutaneous coronary intervention for patients with three-vessel disease: final five-year follow-up of the SYNTAX trial. Eur Heart J 2014;35:2821-30.

18. Fanari Z, Weiss SA, Zhang W, et al. Comparison of percutaneous coronary intervention with drug eluting stents versus coronary artery bypass grafting in patients with multivessel coronary artery disease: meta-analysis of six randomized controlled trials. Cardiovasc Revasc Med 2015;16:70-7.
19. Shiomi H, Morimoto T, Furukawa $\mathrm{Y}$, et al. Comparison of five-year outcome of percutaneous coronary intervention with coronary artery bypass grafting in triple-vessel coronary artery disease (from the Coronary Revascularization Demonstrating Outcome Study in Kyoto PCI/CABG Registry Cohort-2). Am J Cardiol 2015;116:59-65.

20. Bravata DM, Gienger AL, McDonald KM, et al. Systematic review: the comparative effectiveness of percutaneous coronary interventions and coronary artery bypass graft surgery. Ann Intern Med 2007;147:703-16.

21. Fitzgibbon GM, Kafka HP, Leach AJ, et al. Coronary bypass graft fate and patient outcome: angiographic follow-up of 5,065 grafts related to survival and reoperation in 1,388 patients during 25 years. J Am Coll Cardiol 1996;28:616-26.

22. Cho KR, Kim J-S, Choi J-S, et al. Serial angiographic follow-up of grafts one year and five years after coronary artery bypass surgery. Eur J Cardiothorac Surg 2006;29:511-16.

23. Collins $\mathrm{P}$, Webb CM, Chong CF, et al. Radial artery versus saphenous vein patency randomized trial: five-year angiographic follow-up. Circulation 2008;117:2859-64.

24. Goldman S, Zadina K, Moritz T, et al. Long-term patency of saphenous vein and left internal mammary artery grafts after coronary artery bypass surgery: results from a Department of Veterans Affairs Cooperative Study. J Am Coll Cardiol 2004;44:2149-56.

25. Serruys PW, Morice M-C, Kappetein AP, et al. Percutaneous coronary intervention versus coronary-artery bypass grafting for severe coronary artery disease. N Engl J Med 2009;360:961-72. 